\title{
Structural System Identification in the Presence of Resonant Non-Structural Appendages
}

\author{
E. Matta, R. Ceravolo, A. De Stefano \\ A. Quattrone and L. Zanotti Fragonara \\ Department of Structural, Geotechnical and Building Engineering \\ Politecnico di Torino, Turin, Italy
}

\begin{abstract}
Accurate finite element (FE) models are required in many applications of civil engineering. Non-structural elements (NSEs) often interfere with the main structure, altering its stiffness and modal signature. Neglecting such interaction in modelling, although a common design practice, may lead to unreliable predictions of future events and biased interpretations of in-field dynamic tests. In the current literature, the role of NSEs in vibration-based structural system identification (SSI) is well documented for NSEs working in parallel (P-NSEs) with the main structure (e.g. masonry infills in buildings, pavements or railings in bridges and footbridges) but is totally unexplored for NSEs working in series (S-NSEs) with the main structure (e.g. non-structural appendages such as chimneys, parapets, tanks, but also partitions and claddings in their out-of-plane modes). Presenting various numerical and experimental case studies, the present paper shows how in-series NSEs, through augmenting the modal model and by chance resonating with some structural mode, might significantly alter the dynamic behaviour of the main structure, and severely invalidate SSI if not properly accounted for while performing modelling and dynamic identification.
\end{abstract}

Keywords: structural system identification, model calibration, modal analysis, nonstructural appendages, tuned vibration absorbers, modelling errors.

\section{Introduction}

Accurate finite element (FE) models are needed in many applications of Civil Engineering, ranging from health monitoring to structural control, from damage detection to structural evaluation and assessment [1]. Model accuracy can be conveniently improved by means of vibration-based structural system identification (SSI), typically consisting in the calibration of the parameters of a baseline FE model (model-updating) with the experimental modal model derived from vibration 
data. The success of SSI is, however, strictly conditional upon the adoption of a proper "model structure" for the baseline model, i.e. generally speaking of an adequate form of the mathematical equations governing the problem and the associated boundary conditions.

In Civil Engineering, a common source of modelling errors is represented by non-structural elements (NSEs). In fact, NSEs often interfere with the main structure, altering its stiffness and consequently its modal signature. Neglecting such interaction at the modelling stage, although a common design practice, may lead to unreliable predictions of future events as well as to biased interpretations of in-field dynamic tests. Simplifying, this interaction may alternatively obey two main paradigms, according as NSEs work either in parallel (P-NSEs) or in series (SNSEs) with the main structure. P-NSEs (e.g. masonry infills in building structures, pavements or railings in bridges and footbridges), enter the stiffness matrix of the FE model as a mere additive term, modifying frequencies and modeshapes without affecting the model order. A schematic representation of a P-NSE is reported in Figure 1(b). S-NSEs (e.g. non-structural appendages like chimneys, parapets, tanks, ornamentations, but also partitions and facades in their out-of-plane modes) introduce further degrees-of-freedom into the FE description, augmenting the modal model and possibly inducing local/global modal-coupling effects if the appendages happen to be in resonance with some structural mode, until generating a significantly different, new modal system. A schematic representation of an S-NSE is reported in Figure 1(c).

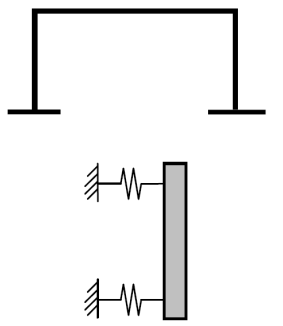

(a)
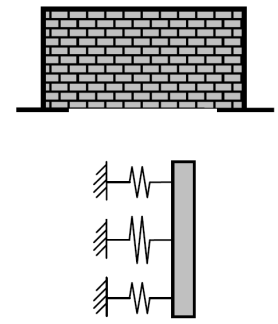

(b)
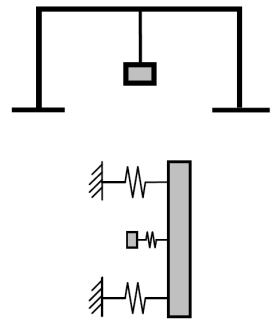

(c)

Figure 1: NSEs on an SDOF structure: (a) main structure (MS); (b) MS with a PNSE; (c) MS with an S-NSE.

Looking at the literature, the role of NSEs in vibration-based structural system identification (SSI) appears generally well documented for P-NSEs [2-11] but totally unexplored for S-NSEs.

In the present paper, numerical and experimental case studies are analysed to demonstrate the influence of "resonant" appendages on the dynamics of the main structure and the need to properly account for them when performing the two steps of modal identification and model calibration. At first, the basic principles of local/global modal-coupling between the main structure and the resonant elements are illustrated for in-series appendages whose frequency is close to the frequency of some structural mode, and the implications of neglecting such modal-coupling effect during modelling are numerically exemplified. Then, numerical simulations are 
produced to show that: (i) if modal-coupling is not correctly identified, the successive step of model-updating may be severely invalidated; (ii) if modalcoupling is properly recognized, two successful approaches can be alternatively followed, respectively based on omitting or including the appendages into the FE model structure. These findings are finally confirmed on a real case study, namely the experimental SSI of a large-scale laboratory building structure containing SNSEs by chance resonant with a structural mode.

\section{Influence of NSEs on the modal and time response of the main structure}

\subsection{Basic modelling configurations for P-NSEs and S-NSEs}

Consider, for simplicity, a shear-type, 2-dimensional (planar), $\mathrm{N}$-storey building structure. Assume, without loss of generality, $N=3$ and modal damping $\zeta=2 \%$ for all the modes.

Four basic configurations are of interest (Figure 2), respectively denoted as:

- $\mathrm{C}_{0}$, corresponding to the main structure (MS) alone (Figure 2a);

- $\mathrm{C}_{1}$, corresponding to the MS with one or more P-NSEs (Figure 2b);

- $\mathrm{C}_{2}$, corresponding to the MS with one or more S-NSEs in the shape of pendulous masses (Figure 2c);

- $\mathrm{C}_{3}$, corresponding to the MS with one or more S-NSEs in the shape of out-ofplane infills (Figure 2d).

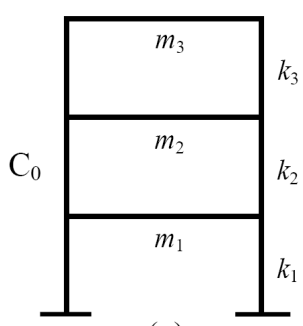

(a)

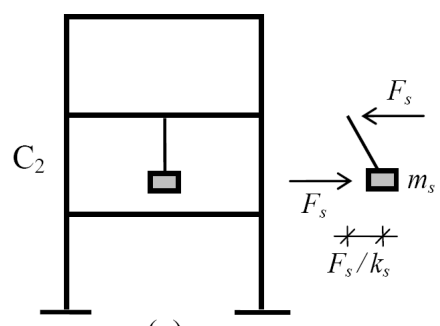

(c)

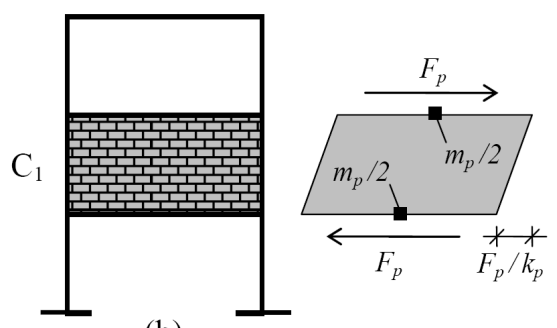

(b)

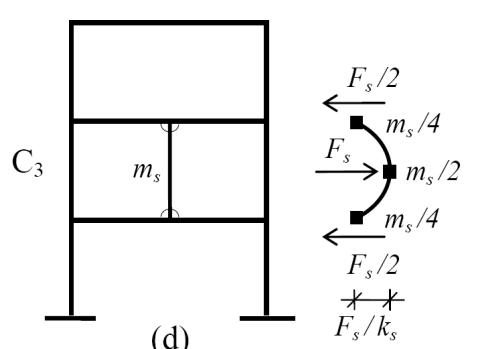

(d)

Figure 2: NSEs on a shear-type 3-storey building structure: (a) MS; (b) MS with a P-NSE (in-plane infill); (c) MS with an S-NSE (pendulous mass);

(d) MS with an S-NSE (out-of-plane infill). 
In order to show the influence of NSEs on the main structure and particularly the implications of an erroneous modelling of NSEs, in the next pages the three configurations $C_{1}, C_{2}$ and $C_{3}$ will be modelled either correctly or incorrectly, in the latter case assuming that the NSEs do not modify the structural stiffness matrix (which remains the same as for $C_{0}$ ) but only affect the mass matrix, with the masses of the NSEs being simply incorporated into the masses of the adjacent floors.

\subsection{Influence of NSEs on the structural modal response}

In order to show the implications of an erroneous modelling of NSEs on the modal response of the building, Figure 3 compares its correct (thick lines) and incorrect (grey areas) transfer functions from the ground acceleration to the maximum (absolute) structural acceleration. For simplicity, the main structure (configuration $\mathrm{C}_{0}$, not depicted in the figure) is chosen to have a fundamental frequency $f_{1}=1.000$ $\mathrm{Hz}$, with its second and third frequencies being then $f_{2}=2.8019 \mathrm{~Hz}$ and $f_{3}=4.0489$ Hz. In particular, the following six cases are reported, each presented in a different sub-figure:

- Case (a): a single in-parallel infill $\left(\mathrm{C}_{1}\right)$ between the $1^{\text {st }}$ and $2^{\text {nd }}$ floors having mass equal to $5 \%$ the total structural mass and stiffness equal to three times the inter-storey structural stiffness (i.e. $\mu=m_{p} / \Sigma m_{i}=5 \% ; k_{p}=\Sigma k_{i}$ ).

- Case (b): a single in-series pendulum $\left(\mathrm{C}_{2}\right)$ attached to the $2^{\text {nd }}$ floor having mass equal to $2.5 \%$ the total structural mass and a frequency equal to the second structural frequency (i.e. $\mu=m_{s} / \Sigma m_{i}=2.5 \% ; f_{s}=f_{2}$ ).

- Case (c): a single in-series infill $\left(\mathrm{C}_{3}\right)$ between the $1^{\text {st }}$ and $2^{\text {nd }}$ floors having mass equal to $5 \%$ the total structural mass and frequency equal to the second structural frequency (i.e. $\mu=m_{s} / \Sigma m_{i}=5 \% ; f_{s}=f_{2}$ ).

- Case (d): three in-parallel infills $\left(\mathrm{C}_{1}\right)$, one at each inter-storey, having total mass equal to $5 \%$ the total structural mass, and each one having stiffness equal to the inter-storey structural stiffness (i.e. $\mu=m_{p i} / m_{i}=5 \% ; k_{p i}=k_{i}$ ).

- Case (e): three in-series pendulums $\left(\mathrm{C}_{2}\right)$, one attached at each floor, having total mass equal to $2.5 \%$ the total structural mass, and having frequencies equal to, respectively, 0.95 ( $1^{\text {st }}$ floor $), 1.00$ ( $2^{\text {nd }}$ floor $)$, and 1.05 ( $3^{\text {rd }}$ floor $)$ times the first structural frequency (i.e. $\mu=m_{s i} / m_{i}=2.5 \% ; f_{s 1}=0.95 f_{1} ; f_{s 2}=$ $\left.1.00 f_{1} ; f_{s 3}=1.05 f_{1}\right)$.

- Case (f): three in-series infills $\left(\mathrm{C}_{3}\right)$, one at each inter-storey, having total mass equal to $5 \%$ the total structural mass, and having frequencies equal to, respectively, 0.95 ( $1^{\text {st }}$ floor $), 1.00$ ( $2^{\text {nd }}$ floor $)$, and 1.05 ( $3^{\text {rd }}$ floor $)$ times the first structural frequency (i.e. $\mu=m_{s i} / m_{i}=5 \% ; f_{s 1}=0.95 f_{1} ; f_{s 2}=1.00 f_{1} ; f_{s 3}=1.05 f_{1}$ ).

Figures 3(a) and 3(d) show the obvious effect of P-NSEs being inserted into the MS, resulting in an increase of the natural frequencies, more or less generalized according to the number and location of the P-NSEs. The dynamic behaviour proves heavily modified, yet the modal order is unaltered (still three modes). This phenomenon is well-known and relatively easily recognizable in SSI.

Figures 3(b), 3(c), 3(e) and 3(f) show the effect of S-NSEs being inserted into the MS, resulting in the generation of new modes, one for each appendage, in the 
vicinity of the original structural modes, as a result of a global-local modal interaction, while the structural modes whose frequencies lay far from the S-NSEs' frequencies are basically unaltered. This phenomenon, quite overlooked by the literature, may significantly bias SSI if not adequately recognized.
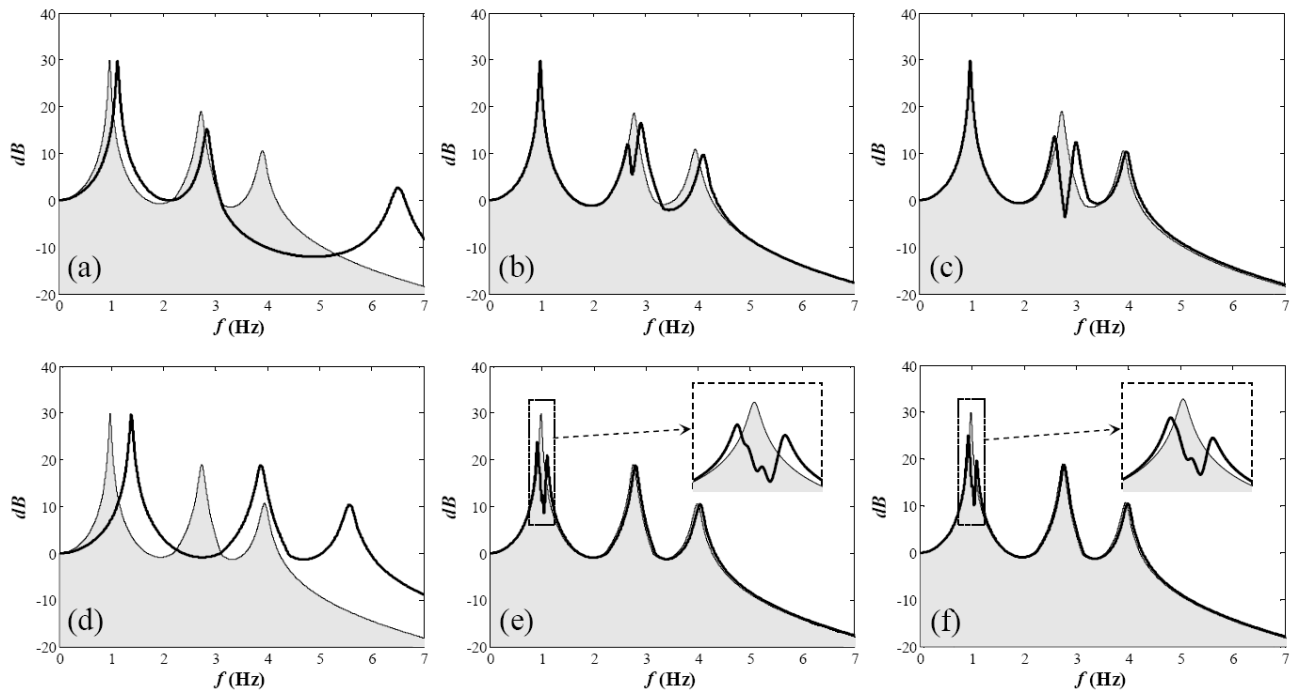

Figure 3: Input-output transfer functions for a shear-type 3-storey building with different arrangements of NSEs (Cases (a) to (f) in the six sub-figures), either correctly modelled (thick lines) or incorrectly modelled (grey areas).

To better understand the modal consequences of such interaction, please refer to Figure 4, where natural frequencies and modeshapes are reported for Case (c) only. Results obtained using the correct modelling are plotted in coloured marks (placed at the DOFs of the FE model) connected by dotted lines; results obtained using the incorrect modelling are plotted in continuous lines. While the incorrect modelling leads to a 3-DOFs FE model possessing three modes only, the correct modelling leads to a 4-DOFs FE model possessing four modes. For clarity of comparison, of these four modes the lowest one and the highest one are still denoted as, respectively, mode 1 and mode 3 (i.e. using the same notation as for the bare MS and as for the incorrect modelling assumption), while the two intermediate modes, born from the interaction of the second structural mode with the S-NSE, are denoted as mode $2 \mathrm{a}$ and mode $2 \mathrm{~b}$. Please note that modes 1 and 3 are basically unmodified by the presence of the S-NSE (the frequency ratios are far from unit), no matter if a correct or incorrect modelling is performed (a slight decrease with respect to the frequencies of the MS, respectively equal to $1 \mathrm{~Hz}$ and $4.0489 \mathrm{~Hz}$, is observed in both cases, due to the NSE increasing the total building mass). Mode 2, instead, as far as the S-NSE is correctly modelled, actually consists of two distinct modes, whose frequencies are respectively lower $\left(f_{2 \mathrm{a}}=2.604 \mathrm{~Hz}\right)$ and higher $\left(f_{2 \mathrm{~b}}=3.005\right)$ than the original frequency of the bare structure (equal to $2.8019 \mathrm{~Hz}$ ), whereas, as far as the S-NSE is modelled only as a rigid mass connected to the floors, it continues to be a single mode with a natural frequency slightly less than the original frequency of the 
bare structure. Please also notice that, as far as no sensor is placed on the NSE, the modeshapes $2 \mathrm{a}$ and $2 \mathrm{~b}$ of the correct model are virtually indistinguishable from each other as well as from the modeshape 2 of the incorrect model and from the modeshape 2 of the bare MS.

It is evident that an erroneous modelling of the S-NSE into the baseline FE model will severely invalidate any attempts to a physically meaningful reconciliation of the model with the experimentally identified modal model. Also, that an inaccurate modal identification, perhaps biased by the analyst's expectation of a three-modes structure, will lead to accept either mode $f_{2 \mathrm{a}}$ or mode $f_{2 \mathrm{~b}}$ as the second mode, with even heavier consequences on the correctness of SSI.

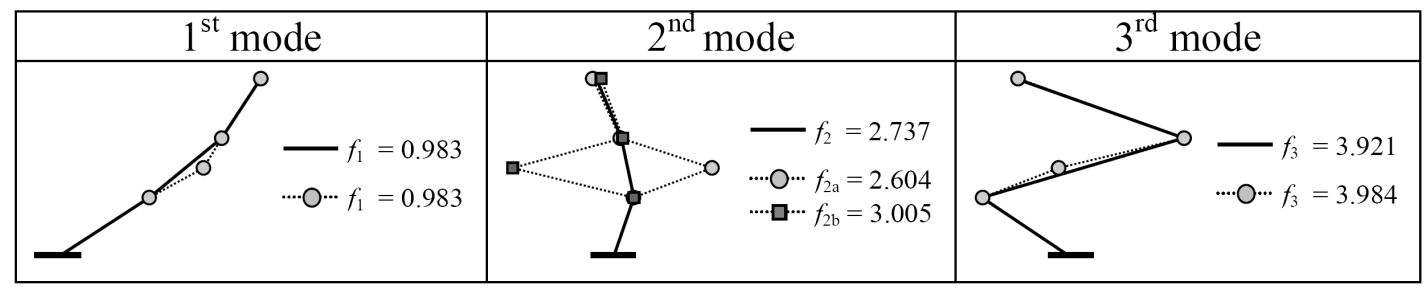

Figure 4: Natural frequencies and modeshapes for Case (c): correct modelling (marks) vs. incorrect modelling (continuous lines).

\subsection{Influence of NSEs on the structural time response}

In order to show the influence of S-NSEs on the time response of the main structure and particularly the implications of an erroneous modelling of S-NSEs, Figure 5 plots the acceleration time-histories at the 3rd floor under two historical seismic records derived from the PEER-NGA Strong Motion Database, in both cases of correct (black line) and incorrect (grey line) modelling of the S-NSEs.

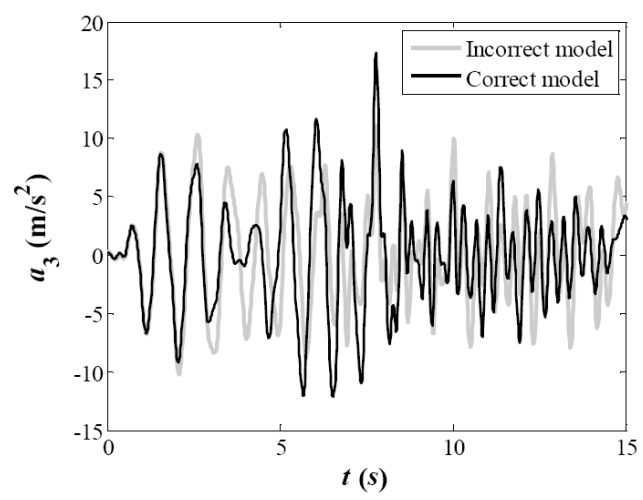

(a)

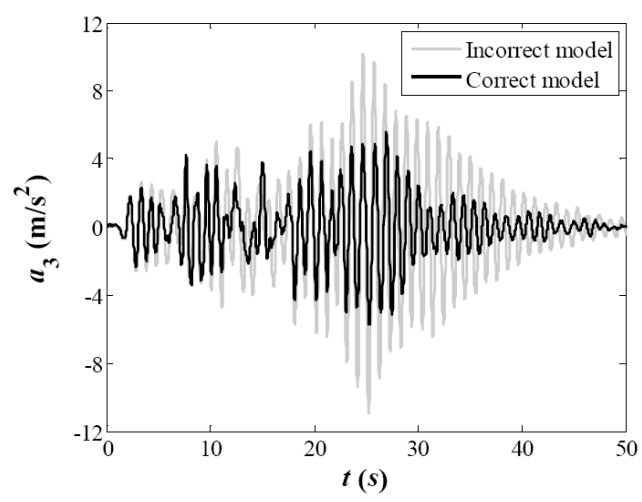

(b)

Figure 5: Simulation of the 3rd floor acceleration under real seismic records for, respectively, correctly and incorrectly modelled NSEs: (a) Case (e) - Record \# 0143

TAB-LN (Tabas); Case (f) - Record \# 1529 TCU102-N (Chi-Chi). 
Figure 5(a) describes Case (e) under Record \# 0143 TAB-LN (Tabas earthquake, 09/16/78). Figure 5(b) describes Case (f) under Record \# 1529 TCU102-N (Chi-Chi earthquake, 09/20/99). It is evident that an incorrect modelling of the S-NSE may have significant effects not only in terms of modal response but also in terms of time response.

\section{SSI in the presence of resonant NSEs: numerical case study}

This section will show the implications of resonant NSEs on modal identification and model-calibration of structural systems. This will be accomplished by recurring to a simple numerical simulation performed on the shear-type MS already introduced in Section 2, in the arrangement denoted as Case (c). This will represent the real structural system.

Suppose that an incorrect shear-type baseline model is initially available for the MS, characterized by three uncertain stiffness parameters, $k_{1}, k_{2}$ and $k_{3}$, and by structural masses exactly known. The (out-of-plane) masonry infill between storeys 1 and 2 is modelled as merely a mass, half of which connected to the $1^{\text {st }}$ storey and the other half to the $2^{\text {nd }}$ storey, whose value is again admitted to be exactly known.

Assume that structural vibration is measured through accelerometers placed at the three storeys (no available accelerometer on the NSE), and that modal identification is performed in order to calibrate the baseline model. For simplicity, no noise is introduced to contaminate modal data.

Various situations may occur, mainly depending on: (i) the correctness of the performed modal identification, (iii) the importance of achieving modelling accuracy, and (ii) the analyst's skills. Three main distinct approaches to the problem can be finally recognized, briefly sketched as follows.

\subsection{Approach 1 (wrong identification and wrong model)}

Assume that the influence of the S-NSE is not understood by the analyst who, expecting a 3-DOF system (according to the incorrect baseline model), looks for three structural modes and, perhaps deceived by measurement noise, identifies them as, let's say, $f_{1}, f_{2 \mathrm{a}}$ and $f_{3}$ (or, equivalently, as $f_{1}, f_{2 \mathrm{~b}}$ and $f_{3}$ ). Using the wrong 3 -DOF model, the analyst calibrates the three stiffness parameters, $k_{1}, k_{2}, k_{3}$, so that the modelled frequencies equal the identified frequencies. Note that he might have used modeshapes too to define the error function (naturally defined only at the floors) but, regarding them as less accurate than frequencies and assuming the problem as determinate ( 3 data and 3 unknowns), he chooses to only rely on frequencies in this case. Anyway, Figure 4 suggests that, due to the similarity of correct and incorrect modeshapes (unless at the unmeasured NSE), using modeshapes as updating parameters would have scarcely affected the results. 


\subsection{Approach 2 (correct identification but wrong model)}

The influence of the NSE is understood by the analyst who, identifying four real modes and attributing modes $2 \mathrm{a}$ and $2 \mathrm{~b}$ to the tuning of the S-NSE with the second structural mode, recognizes that the 3-DOF model is inadequate to reproduce them all. However, being not interested in an accurate modelling of the NSE and of the second mode, he considers it an unnecessary cost to modify the model structure to include the NSE, and decides to keep the baseline model structure unaltered (incorrect modelling).

Since the four identified modes are incompatible with the three simulated modes, he excludes the second mode from the experimental data, and calibrates the model on the bases of the first and third models only. Since, however, two frequencies are insufficient to calibrate three stiffness parameters, he uses modeshapes too in order to avoid indeterminateness of the problem (i.e. non-uniqueness of the solution), for instance both modeshapes 1 and 3. If no modeshapes were available from the identification, of course, this approach would not be viable unless the analyst could reduce the number of unknowns by making some further a priori assumption.

\subsection{Approach 3 (correct identification and correct model)}

The influence of the NSE is understood by the analyst who, based on the correct identification of the four real modes, accepts to improve the model so as to account for the S-NSE. Still assuming the masses as completely known, he therefore updates the four unknown stiffness parameters, $k_{1}, k_{2}, k_{3}$ and $k_{\text {s }}$, (see again Figure $2(\mathrm{~d})$ ) using the four identified frequencies.

\subsection{Comparison of the three approaches}

The three approaches described above are obviously only some of the possible paths an analyst might follow to perform SSI in the presence of S-NSEs. For instance, he might use redundant information to increase robustness (instead of merely avoiding indeterminateness). Or, after recognizing the importance of the NSE (Approaches 2 and 3), he might decide to repeat measurements and calibration after deploying additional sensors on the NSE as well. Anyway, in their simplicity, they have the merit, on the one hand, to indicate two alternative viable strategies for dealing with S-NSEs in SSI (Approaches 2 and 3) and, on the other hand, to show the drawbacks of not following them (Approach 1).

The results obtained through applying the three approaches to the simple numerical case study under consideration are summarized in Table 1. The Approach 1 is presented twice, according as either $f_{2 a}$ (Approach 1-a) or $f_{2 b}$ (Approach 1-b) are chosen for updating. In particular, Table 1 presents the optimal solutions of the model-updating problem, obtained, for each approach, by finding the modelupdating normalized parameters $\bar{k}_{i}$ (where normalization is done by dividing the dimensional parameters by their nominal value, here assumed equal to the true value) which numerically minimize the following objective function: 


$$
f_{o b}=\sqrt{\frac{\sum_{i}\left(f_{i} / f_{e i}-1\right)^{2}+\lambda^{2} \sum_{j}\left\|\phi_{j}-\phi_{e j}\right\|^{2}}{\sum_{i}+\lambda^{2} \sum_{j}}}
$$

in which the first summation (subscript $i$ ) refers to the frequency residual and the second summation (subscript $j$ ) refers to the mode-shape residual, $f_{i}$ and $f_{e i}$ being, respectively, the analytical and the experimental natural frequencies, $\phi_{j}$ and $\phi_{e j}$ being the analytical and the experimental mode-shapes (normalized so as to have unit norm), $\left\|\phi_{j}-\phi_{e j}\right\|=\sqrt{2\left[1-\sqrt{\operatorname{MAC}\left(\phi_{j}, \phi_{e j}\right)}\right]}$ being the 2-norm (or Euclidean distance) between vectors $\phi_{j}$ and $\phi_{e j}$, and $\lambda$ being the relative weight assigned to the modeshape residual with respect to the frequency residual, accounting for the different reliability of mode-shape information with respect to frequency information (and in the present example taking as equal to 1). Please notice that the analyst may freely choose to include into Eq.(1) only a selected subset of the available frequencies (subscript $i$ ) and mode-shapes (subscript $j$ ), with the frequency subset and the modeshapes subset not necessarily being the same, while in the particular case when the two subsets are the same Eq.(1) simplifies as follows:

$$
f_{o b}=\sqrt{\frac{\sum_{i}\left[\left(f_{i} / f_{e i}-1\right)^{2}+\lambda^{2}\left\|\phi_{i}-\phi_{e i}\right\|^{2}\right]}{\sum_{i}\left(1+\lambda^{2}\right)}}
$$

Eq.(1) and Eq.(2) are so conceived that when all residuals equal a certain value, then $f_{o b}$ equals the same value. In this sense, $f_{o b}$ is the weighted error of the analytical modal model with respect to the experimental modal model.

Beyond the identified (normalized) updating-parameters, Table 1 presents, in the last column, the RMS (root mean square) distance, $d$, between them and their true value (1.000 in the present case), taken as a measure of the calibration error. The value of the objective function, $f_{o b}$, is not reported in Table 1 since nearly null in the present example.

\begin{tabular}{cccccc}
\hline Approach & $\bar{k}_{1}$ & $\bar{k}_{2}$ & $\bar{k}_{3}$ & $\bar{k}_{s}$ & $d(\%)$ \\
\hline $1-\mathrm{a}$ & 0.948 & 1.159 & 0.850 & n.a. & 13.0 \\
$1-\mathrm{b}$ & 1.350 & 0.685 & 1.343 & n.a. & 33.6 \\
2 & 0.985 & 1.045 & 1.004 & n.a. & 2.7 \\
3 & 1.000 & 1.000 & 1.000 & 1.000 & 0.0 \\
\hline
\end{tabular}

Table 1: Model calibration for the three approaches. 
Table 1 clearly shows that mistaking the modal identification phase (Approach 1) may lead to unacceptable errors in the parameter domain ( $d$ up to 33.6\%). Understanding the real "modal structure" without correcting the baseline FE "model structure" (Approach 2) may lead to still acceptable calibration errors (2.4\%). The correct identification and the correct modelling (Approach 3) are obviously the best option, in the present ideal case leading to a null error in the parameter domain as a consequence of the total absence of errors in the identified modal data and in the model structure.

\section{SSI in the presence of resonant NSEs: an experimental case study}

The present section will show the influence of resonant S-NSEs on SSI by means of an experimental case-study.

\subsection{The structure}

The test structure is a large-scale (2:3) model of a two-storey steel frame building with composite steel-concrete floors (Figure 6(a)). The steel structure, consisting of columns and beams orthogonally interconnected into a regular (doubly-symmetrical) three-dimensional frame with one bay in both directions and two rectangular floors (level 1 and 2), is mounted on a rigid horizontal base (level 0), resting on two sliding guides and connected to a dynamic hydraulic actuator which can impart the desired mono-dimensional excitation to the structure. Four HE140B equal columns, fixed to the base, extend continuously to the top floor. Eight IPE180 lateral beams, welded to the columns, support the two composite floors, made up of concrete slabs cast on coffer profiled steel sheeting. The columns free length is $4.00 \mathrm{~m}$, divided into two $2.00 \mathrm{~m}$ inter-storey heights. The beams length is $4.00 \mathrm{~m}$ in the along-excitation (longitudinal) direction and $3.00 \mathrm{~m}$ in the across-excitation (transverse) direction. The floors thickness is larger than expected because of the sagging effect occurred during concrete casting. In order to house the dissipating devices during tests on the controlled structure, four $\mathrm{V}$-inverted braces, crowned with gusset plates, are bolted at both storeys, parallel to the longitudinal direction.

At the initial stage of the Project, the need of an accurate numerical model of the benchmark building motivated a preliminary campaign of dynamic tests on the uncontrolled structure (i.e. with no dissipative device installed). In order to increase the amount of data available, classical "perturbed boundary condition testing" was pursued, consisting in perturbing both the structure and the analytical model by adding the same amount of mass at given positions. A total of eight concrete blocks (about $340 \mathrm{~kg}$ each) were fixed onto the floors during testing according to three different configurations. Starting from the basic configuration (BC), characterized by no additional mass, a second doubly-symmetric configuration (SC) was obtained by the addition of four blocks on each storey, then a non-symmetric configuration (NC) was obtained removing two blocks from the SC configuration at each storey. 
For each of the three mass configurations, ambient vibration tests were conducted on the uncontrolled structure, keeping the sliding guides locked. The dynamic response was measured by 15 accelerometers deployed in the most significant observation points. The location of the accelerometers and of the concrete blocks is described in Figure 6(b). Please notice that no sensors are placed on the upper braces.

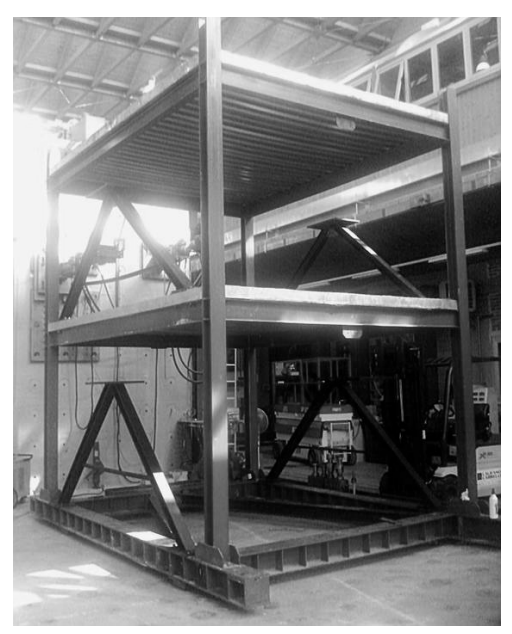

(a)
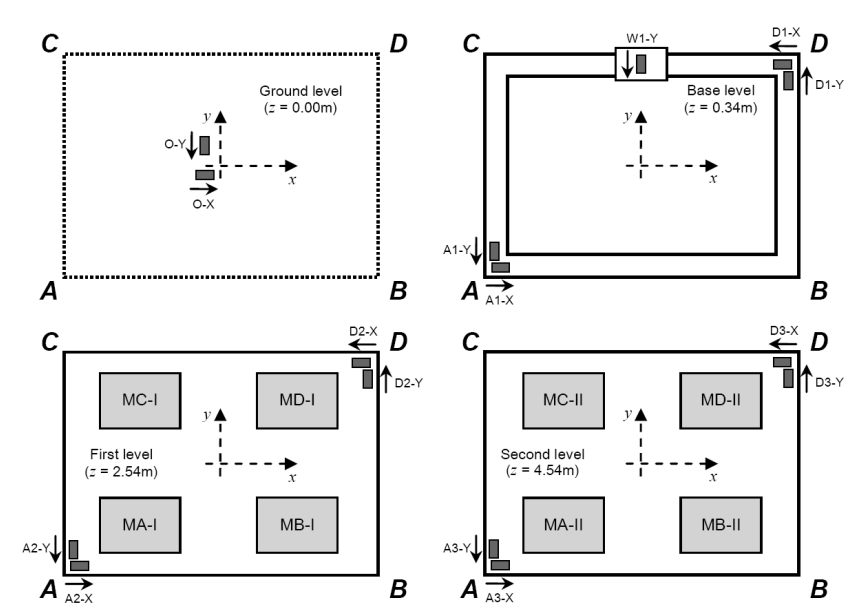

(b)

Figure 6: The structural prototype: (a) overall view; (b) location of accelerometers and additional blocks.

\subsection{The initial FE model (NSEs neglected)}

The FE model developed at the initial stages of the project represented the main structure alone, with no consideration for the dynamics of the NSEs, merely included as rigid masses into the masses of the floors.

This kind of model, reasonable until the importance of the NSEs' dynamics was understood, will be taken as representative of the incorrect modelling of S-NSEs, in the sense explained in the previous Section.

According to this model, stiffness and mass matrices are statically condensed to the six translational and rotational displacement components of the first and second floors' geometrical centres, included in the vector $\eta=\left\{\begin{array}{llllll}d_{x 1} & d_{y 1} & d_{\theta 1} & d_{x 2} & d_{x 2} & d_{\theta 2}\end{array}\right\}^{\mathrm{T}}$, under the hypotheses of axial rigidity of Euler-Bernoulli type columns and beams, in-plane rigidity of floor slabs, and lumped mass formulation for columns and beams. Columns are clamped at their base (i.e.: no dynamics attributed to the base level), the $\mathrm{V}$-inverted braces deprived of any dynamics of their own, and no connection is explicitly recognized between the floor slab and the steel beams. Each of the additional concrete blocks is accounted for as a translational inertia, and enters the system mass matrix with its own mass, static moment and polar inertia. With these assumptions, for each mass configuration the nominal (reference) FE model is obtained through equalling each geometrical and mechanical parameter to its expected (nominal) value. 


\subsection{The experimental modal model}

The experimental modal model is identified on the basis of ambient vibration tests. Three classical output-only methods are used to extract frequencies and modeshapes, respectively working in the time-domain, in the frequency-domain and in the timefrequency domain: ERA [12], FDD [13] and TFIE [14].

The details of the experimental modal analysis are not reported here for brevity's sake. Two results are, however, worth mentioning. The first, well expected, is the progressive reduction of all the natural frequencies with increasing additional masses on the structure, and a slight modification of the modeshapes too (with the loss of their double-symmetry in the NS configuration). The second, which was totally unexpected, is the dynamic interaction (due to frequency closeness) between the out-of-plane mode of the $\mathrm{V}$-inverted braces attached to the first storey and the fourth mode (i.e. the second flexural mode in the transverse direction) of the main frame [15]. Such interaction explains the existence of seven structural modes instead of the expected six ones, the modes $4 \mathrm{a}$ and $4 \mathrm{~b}$ having distinct frequencies but nearly undistinguishable modeshape components at both stories. The 6-DOF analytical model is obviously inadequate to simulate such modal coupling effect.

\subsection{The augmented FE model (NSEs included)}

The initial FE model is augmented to include the presence of NSEs. This is done by incorporating in the original 6-DOFs model one additional DOF corresponding to the transverse (Y-) displacement of the pair of upper braces. In practice, a SDOF appendage is connected to the first storey representing the dynamics of both $\mathrm{V}$ inverted braces. The nominal value of the mass of this appendage is assumed to be twice the effective mass of each V-inverted brace (the latter being estimated as the sum of the mass of the gusset plate and half the mass of the inclined braces), and namely equal to $70 \mathrm{~kg}$. The frequency of the appendage is assumed to be equal to 10 $\mathrm{Hz}$, as suggested by the instrumented lower brace and by the auto-spectral densities plotted in the along-Y direction [15].

\subsection{Model calibration according to Approaches 1 to 3}

The three Approaches already presented in Section 3 are here repeated for the real case study. Since, however, in this case the true solution is not available, caution must be placed in the correct selection of the updating parameters, and a multimodel updating procedure is implemented to enhance robustness.

According to this procedure, several distinct sets of updating parameters are selected by the analyst, and updating is repeated for each case [16]. First of all, the overall set of (normalized) updating parameters is chosen, using a priori knowledge and sensitivity analysis (Figure 7). 


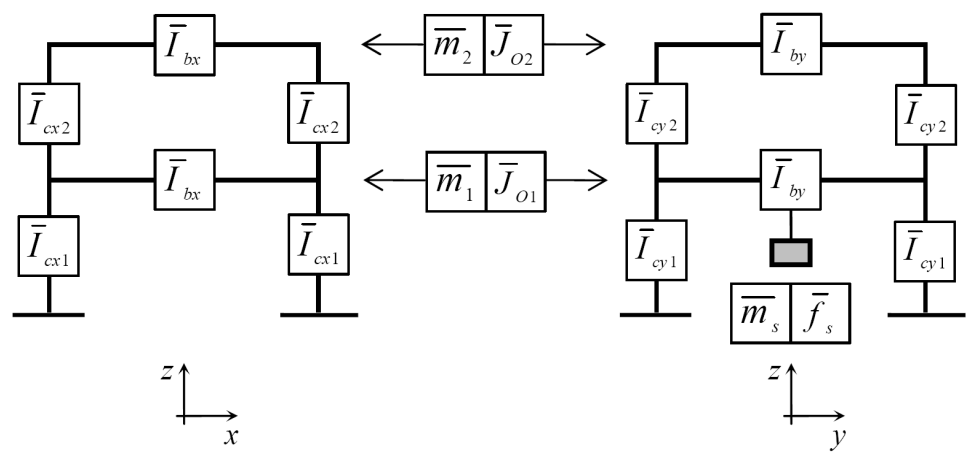

Figure 7: The FE model with the 12 potential (normalized) updating parameters.

Then, three different sets of updating parameters are selected for each of the three Approaches, denoted as $\mathrm{M}_{1}, \mathrm{M}_{2}$ and $\mathrm{M}_{3}$ for the Approaches 1 and 2 (initial incorrect model), and as $\mathrm{M}_{1+}, \mathrm{M}_{2+}$ and $\mathrm{M}_{3+}$, due to the inclusion of NSEs, for the Approach 3 (augmented correct model).

Results are reported in Tables 2 to 5. Please notice that different objective functions are used for each approach, depending on the number of modes (6 modes for the Approach 1, 5 modes for the Approach 2, 7 modes for the Approach 3).

\begin{tabular}{cccccccccccccc}
\hline Model & $\bar{I}_{c x 1}$ & $\bar{I}_{c y 1}$ & $\bar{I}_{c x 2}$ & $\bar{I}_{c y 2}$ & $\bar{I}_{b x}$ & $\bar{I}_{b y}$ & $\bar{m}_{1}$ & $\bar{m}_{2}$ & $\bar{J}_{O 1}$ & $\bar{J}_{O 2}$ & $\bar{m}_{s}$ & $\bar{f}_{s}$ & $f_{o b 1 a}(\%)$ \\
\hline $\mathrm{M}_{1}$ & .952 & .928 & 1 & 1 & 1 & 1 & 1.045 & .794 & .946 & .793 & n.a. & n.a. & 1.26 \\
$\mathrm{M}_{2}$ & .881 & .913 & 1.099 & 0.989 & 1 & 1 & 1.041 & .774 & .944 & .766 & n.a. & n.a. & 1.23 \\
$\mathrm{M}_{3}$ & .952 & .857 & 1 & 1 & .984 & 1.137 & 1.035 & .789 & .928 & .804 & n.a. & n.a. & 1.24 \\
\hline
\end{tabular}

Table 2: Approach 1-a.

\begin{tabular}{cccccccccccccc}
\hline Model & $\bar{I}_{c x 1}$ & $\bar{I}_{c y 1}$ & $\bar{I}_{c x 2}$ & $\bar{I}_{c y 2}$ & $\bar{I}_{b x}$ & $\bar{I}_{b y}$ & $\bar{m}_{1}$ & $\bar{m}_{2}$ & $\bar{J}_{O 1}$ & $\bar{J}_{O 2}$ & $\bar{m}_{s}$ & $\bar{f}_{s}$ & $f_{o b 1 b}(\%)$ \\
\hline $\mathrm{M}_{1}$ & 0.451 & 0.658 & 1 & 1 & 1 & 1 & .528 & .628 & 0.666 & .515 & n.a. & n.a. & 4.89 \\
$\mathrm{M}_{2}$ & 1.172 & 0.825 & .732 & 2.510 & 1 & 1 & .973 & .870 & 1.226 & .933 & n.a. & n.a. & 2.31 \\
$\mathrm{M}_{3}$ & 0.820 & 2.873 & 1 & 1 & 1.155 & .395 & .960 & .837 & 1.221 & .812 & n.a. & n.a. & 2.10 \\
\hline
\end{tabular}

Table 3: Approach 1-b.

\begin{tabular}{cccccccccccccc}
\hline Model & $\bar{I}_{c x 1}$ & $\bar{I}_{c y 1}$ & $\bar{I}_{c x 2}$ & $\bar{I}_{c y 2}$ & $\bar{I}_{b x}$ & $\bar{I}_{b y}$ & $\bar{m}_{1}$ & $\bar{m}_{2}$ & $\bar{J}_{O 1}$ & $\bar{J}_{O 2}$ & $\bar{m}_{s}$ & $\bar{f}_{s}$ & $f_{o b 2}(\%)$ \\
\hline $\mathrm{M}_{1}$ & .756 & .792 & 1 & 1 & 1 & 1 & .887 & .716 & .830 & .694 & n.a. & n.a. & .856 \\
$\mathrm{M}_{2}$ & .782 & .778 & .915 & 1.073 & 1 & 1 & .862 & .726 & .834 & .707 & n.a. & n.a. & .843 \\
$\mathrm{M}_{3}$ & .629 & .755 & 1 & 1 & 1.254 & 1.192 & .816 & .777 & .790 & .740 & n.a. & n.a. & .780 \\
\hline
\end{tabular}

Table 4: Approach 2. 


\begin{tabular}{cccccccccccccc}
\hline Model & $\bar{I}_{c x 1}$ & $\bar{I}_{c y 1}$ & $\bar{I}_{c x 2}$ & $\bar{I}_{c y 2}$ & $\bar{I}_{b x}$ & $\bar{I}_{b y}$ & $\bar{m}_{1}$ & $\bar{m}_{2}$ & $\bar{J}_{O 1}$ & $\bar{J}_{O 2}$ & $\bar{m}_{s}$ & $\bar{f}_{s}$ & $f_{o b 3}(\%)$ \\
\hline $\mathrm{M}_{1+}$ & .711 & .772 & 1 & 1 & 1 & 1 & .839 & .705 & .807 & .673 & 1.025 & 1.020 & .839 \\
$\mathrm{M}_{2+}$ & .806 & .788 & .836 & 1.028 & 1 & 1 & .830 & .735 & .800 & .716 & 1.084 & 1.013 & .739 \\
$\mathrm{M}_{3+}$ & .607 & .769 & 1 & 1 & 1.305 & 1.163 & .803 & .785 & .785 & .742 & 1.062 & 1.011 & .659 \\
\hline
\end{tabular}

Table 5: Approach 3.

In order to genuinely capture improvements in the model, the multiple solutions must be compared in terms not only of their fitting capability but also of the plausibility of the underlying parametric description. Using, as in Table 1, the RMS error to measure the distance in the parameter domain between different solutions, it results that the distance from the best model $\left(\mathrm{M}_{3+}\right)$ equals $17.6 \%$ for $\mathrm{M}_{3}$ according to the Approach 1a, $72.9 \%$ for $\mathrm{M}_{3}$ according to the Approach $1 \mathrm{~b}$, and only $2.1 \%$ for $\mathrm{M}_{3}$ according to the Approach 2.

\subsection{Discussion of the results}

Looking at the results obtained in the previous subsection the following considerations can be formulated.

First of all, the best model is $\mathrm{M}_{3+}$ calibrated using the Approach 3. This model is the analogue of the exact model in Section 3, and can be used here as the reference term for evaluating any other solution. This model not only achieves a very good matching of the experimental modal data, but also possesses sound physical meaning, with the stiffness of the lower columns being less than nominal, expectedly due to the imperfect clamp at their base, and the stiffness of the beams being larger than nominal, due to the partial collaboration of the floor slabs.

The same Approach 3 gives not so good results for the updating set $\mathrm{M}_{1_{+}}$and $\mathrm{M}_{2+}$, because both sets exclude the stiffness of the beams from the number of the updating parameters. This result confirms the importance of correctly choosing the set of updating parameters, which can be profitably done recurring to multi-model approaches.

The Approach 2 provides satisfactory results too. The best model, once again $\mathrm{M}_{3}$, is very close to the $\mathrm{M}_{3+}$ model obtained with the Approach 3, both in terms of the objective function and in terms of the distance in the search domain: the RMS distance is only $2.1 \%$ and the maximum distance along a single parameter, $I_{c x 1}$, is only $3.7 \%$. This clearly confirms the validity of the Approach 2 . The calibrated model is very close to the best possible one, with no need to modify the model structure through including the NSE, and the modal matching (with the obvious exception of modes $4 \mathrm{a}$ and $4 \mathrm{~b}$ ) is quite satisfactory in absolute terms.

As for the numerical case-study, instead, the Approach 1 shows all its limitations, demonstrating the significance of NSEs and consequently the need to understand their role during the identification phase. Not only the objective function significantly increases but also the updated parameters diverge significantly from the optimal ones obtained with the model $\mathrm{M}_{3+}$, particularly if $f_{2 b}$ is taken as the structural frequency (Approach 1-b), in which case the RMS distance grows to an 
unacceptable $72.9 \%$ (with an error of $274 \%$ along $I_{c y 1}$ and $-66 \%$ along $I_{b y 1,2}$ ), implying the physical impossibility of the solution.

This result clearly shows the severity of the modelling error consisting in forgetting to model the NSEs. In the present case, luckily, the unsoundness of the solution clearly tells that something has gone wrong. But unfortunately this is not a general rule.

\section{Conclusions}

In this paper, numerical and experimental case studies have been analysed to demonstrate the influence of "resonant" appendages on the dynamics of the main structure and the need to properly account for them when performing the two steps of modal identification and model calibration. At first, the basic principles of local or global modal-coupling between the main structure and the resonant elements have been illustrated for in-series appendages whose frequency is close to the frequency of some structural mode, and the implications of neglecting such modal-coupling effect during modelling have been numerically exemplified. Then, numerical simulations have been produced to show that: (i) if modal-coupling is not correctly identified, the successive step of model-updating may be severely invalidated; (ii) if modal-coupling is properly recognized, two successful approaches can be alternatively followed, respectively based on omitting or including the appendages into the FE model of the structure. These findings have been finally confirmed on a real case study, namely the experimental SSI of a large-scale laboratory building structure containing S-NSEs by chance resonant with a structural mode. Resonant appendages have been finally proven to significantly influence system dynamics, and useful guidelines have been provided to successfully pursue SSI in their presence.

\section{References}

[1] M.I. Friswell, J.E. Mottershead, "Finite Element Model Updating in Structural Dynamics", Kluwer Academic Press, Dordrecht, the Netherlands, 1995.

[2] C.D. Eamon, A.S. Nowak, "Effect of secondary elements on bridge structural system reliability considering moment capacity", Structural Safety, 26, 29-47, 2004.

[3] M.-S. Marefat, E. Ghahremani-Gargary, S. Ataei, "Load test of a plain concrete arch railway bridge of $20-\mathrm{m}$ span", Construction and Building Materials, 18, 661-667, 2004.

[4] J.Y. Kim, E. Yu, D.Y. Kim, S.-D. Kim, "Calibration of analytical models to assess wind-induced acceleration responses of tall buildings in serviceability level", Engineering Structures, 31, 2086-2096, 2009.

[5] J.-A. Goulet, P. Kripakaran, I.F.C. Smith, "Estimation of modelling errors in structural system identification", 4th Int. Conf. on Structural Health Monitoring of Intelligent Infrastructure (SHMII-4), 2009. 
[6] A. Turer, B.M. Shahrooz, "Load rating of concrete-deck-on-steel-stringer bridges using field-calibrated 2D-grid models", Engineering Structures, 33, 1267-1276, 2011.

[7] J.M.W. Browjohn, A.A. Dumanoglu, C.A. Taylor, "Dynamic investigation of a suspension footbridge", Engineering Structures, 16, 395-406, 1994.

[8] S. Zivanovic, A. Pavic, P. Reynolds, "Modal testing and FE model tuning of a lively footbridge structure", Engineering Structures, 28, 857-868, 2006.

[9] P. Reynolds, A. Pavic, M.R. Willford, "Prediction and measurement of stadia dynamic properties", 23rd International Modal Analysis Conference (IMAC XXIII), 2005.

[10] M.A.M. Torkamani, A.K. Armadi, "Stiffness identification of a tall building during construction period using ambient tests", Earthquake Engineering \& Structural Dynamics, 16(8), 1177-1188, 1988.

[11] R.L. Boroschek, H. Baesler, C. Vega, "Experimental evaluation of the dynamic properties of a wharf structure", Engineering Structures, 33, 344-356, 2011.

[12] J.N. Juang, R.S. Pappa, “An eigensystem realisation algorithm (ERA) for modal parameter identification and modal reduction", NASA/JPL Workshop on Identification and Control of Flexible Space Structures, 1984.

[13] R. Brincker, L. Zhang, P. Andersen, "Modal identification of output-only systems using frequency domain decomposition", Smart Materials and Structures, 10, 441-445, 2001.

[14] P. Bonato, R. Ceravolo, A. De Stefano, F. and Molinari, "Use of cross timefrequency estimators for the structural identification in non-stationary conditions and under unknown excitation", Journal of Sound and Vibration, 237, 775-791, 2000.

[15] E. Matta, A. De Stefano, A. Quattrone, "Reliability issues in vibration-based system identification: lessons from the JETPACS case study", 4th Int. Conf. on SHM of Intelligent Infrastructure, Zurich, Switzerland, 2009.

[16] E. Matta, A. De Stefano, "Generating alternatives from multiple models: how to increase robustness in parametric system identification", 5th Int. Conf. on SHM of Intelligent Infrastructure, Cancún, Mexico, 2011. 
$2012-12-31$

\section{Structural system identification in the} presence of resonant non-structural appendages

Matta, Emiliano

Civil-Comp Press

Emiliano Matta, Rosario Ceravolo, Alessandro De Stefano, Antonino Quattrone, Luca Zanotti

Fragonara. Structural system identification in the presence of resonant non-structural appendages. 11th International Conference on Computational Structures Technology (CST2012), 4-7 September 2012, Dubrovnik, Croatia.

https://dspace.lib.cranfield.ac.uk/handle/1826/12257

Downloaded from Cranfield Library Services E-Repository 